\title{
El fracaso del periódico Ahora, el ocaso de la prensa impresa española
}

\author{
Ddo. Pedro Vázquez-Miraz| pedro.vazquez@udc.es
}

Universidad de A Coruña

\author{
Palabras clave \\ comunicación; prensa; periodismo digital; periódicos \\ impresos; ideología periodística \\ Sumario \\ 1. Introducción. 2. Metodología. 3. Resultados. 4. \\ Discusión. 5. Conclusiones. 6. Bibliografía.
}

\section{Resumen}

Se presenta en este artículo una revisión sobre la breve historia y trayectoria del semanario español Ahora, periódico presentado tanto en papel, bajo formato sábana, como en Internet, fundado por Miguel Ángel Aguilar, cuyo primer número a la venta salió a los quioscos de toda España el 18 de septiembre del año 2015, siendo el último de sus números, antes de su cierre, el del día 14 de octubre del 2016. A través de

una revisión hemerográfica dónde se analizan todas las noticias asociadas con la aparición de este nuevo periódico en los demás medios digitales y el propio estudio de los números de esta publicación, se pretende encontrar las causas por las que se produjo la desaparición de este efímero producto, el cual estaba dirigido por notables profesionales de gran prestigio. Las razones por las que se produjo su cierre son evidentes: excesivos costes de producción, precio poco competitivo para un producto novedoso y una limitada campaña de promoción en los medios de comunicación; este fracaso se magnifica si es comparado con el auge (desde 2015) de los nuevos periódicos digitales españoles, pudiéndose concluir que Ahora es la verificación de la nula viabilidad económica de la prensa impresa en España.

\section{Cómo citar este texto:}

Pedro Vázquez-Miraz (2017): "El fracaso del periódico Ahora, el ocaso de la prensa impresa española”, en Miguel Hernández Communication Journal, nº8, pp. 267 a 286. Universidad Miguel Hernández, UMH (Elche-Alicante). Recuperado el _ de de 20__ de: link del artículo en mhjournal.org] 


\title{
The failure of the newspaper Now, the decline of the Spanish print
}

\author{
Ddo. Pedro Vázquez-Miraz| pedro.vazquez@udc.es \\ Universidad de A Coruña
}

\author{
Keywords \\ comunication; press; digital journalism; printed \\ newspapers; journalistic ideology \\ Summary \\ 1. Introduction. 2. Method. 3. Results. 4. \\ Discussion. 5. Conclusions. 6. References.
}

\section{Abstract}

This article presents a review on the brief history and trajectory of the Spanish weekly newspaper Ahora, a newspaper presented both in paper, in broadsheet format and on the Internet, founded by Miguel Ángel Aguilar, whose first number on sale went to spanish kiosks on September 18, 2015, and its last number, before its closure, on October 14, 2016. We analyzed the news associated with the appearance of this new newspaper in the other digital media through a hemerographic review and also studied the numbers of this publication, trying to find the causes for the disappearance of this ephemeral product, which was directed by notable professionals of great prestige. The reasons for the closure of this newspaper are obvious: excessive production costs, uncompetitive price for a new product and a limited promotion campaign in the mass; this failure is magnified if we compare the boom (since 2015) of the new Spanish digital newspapers, so that we can concluded that Ahora is the verification of the null economic viability of the printed press in Spain.

\section{How to cite this text:}

Pedro Vázquez-Miraz (2017): "The failure of the newspaper Now, the decline of the Spanish print”, en Miguel Hernández Communication Journal, no8, pp. 267 to 286. Universidad Miguel Hernández, UMH (Elche-Alicante). Accessed 20_ in: [paper link in mhjournal.org] 


\section{Introducción}

De Aguinaga (2002: 158) define al periodismo como "la presentación social de la industria de la información, que nace, con la empresa periodística, a finales del siglo pasado"; y si bien fue en 1661 cuando se comienza a publicar el que se considera el primer periódico español, La Gaceta Nueva (Alía, 2013: 105), esta industria se consolidó en España como tal en el siglo XIX (Alía, 2013: 105), caracterizándose ese siglo por presentar de forma exponencial un enorme número de periódicos, llegando a haber en el país en el año 1899, 1.347 diarios (Alía, 2013: 105), situación que cambiaría drásticamente ya avanzado el siglo XX, al producirse en el sector una despiadada concentración mediática, pasándose de los 2.289 títulos de 1920 a los 101 periódicos editados en el año 2000 (Alía, 2013: 106).

A principios del siglo XX, la concepción inicial del periodismo español fue evolucionando desde una perspectiva inicial que fomentaba la profesión como un valor didáctico y moralizante que permitía formar a la sociedad, a una visión más pragmática, donde el periódico se considera una empresa más; donde el periodista debe estar más comprometido con la empresa que con la ética (García-Galindo, 2005: 186).

Iniciado el siglo XXI, la industria de los periódicos impresos en España era una de las más importantes del continente europeo, moviendo de forma aproximada una cifra neta de negocio (en el año 2006) de 2.802 millones de euros (Larrañaga, 2009: 64). Los periódicos españoles más importantes gozaban de una aceptable salud económica, particularmente El País, periódico creado en el año 1976, el cual sigue siendo el diario escrito hegemónico en el espectro ideológico del centro-izquierda, y El Mundo (creado en 1989 por Pedro José Ramírez, director de Diario 16 durante el período 1980-1989), orientado al centro-derecha y su rival más próximo; siendo las excepciones en este período un debilitado $A b c$ (de ideología conservadora) que finalmente sería incorporado al Grupo Correo en el año 2001 (Sotelo, 2015: 840) y un recién aparecido diario La Razón (creado en 1998 y de misma ideología que el anterior), fundado por el periodista Luis María Ansón (Nogales y MancinasChávez, 2014: 315), director del $A b c$ en el período 1983-1997.

El siglo XXI vio en sus inicios el ocaso del los grandes grupos mediáticos españoles que dirigían estos periódicos, pues erróneas decisiones empresariales tomadas en el año 2007, antes de la crisis económica [a pesar de que ya se veían cifras de agotamiento en las ventas de los periódicos impresos de Europa, Norteamérica y Oceanía (Larrañaga, 2009: 63)], como la compra por 
parte de Unedisa (grupo editor de El Mundo) del grupo Recoletos (grupo editor de los diarios impresos Marca y Expansión) (Sotelo, 2015: 837), la compra del 100\% de los activos de Sogecable por el Grupo Prisa (grupo editor de El País) (Almirón, 2007) o la compra por parte de Vocento (grupo editor del $A b c$ ) del diario gratuito de papel Qué! (periódico cerrado en el año 2012 [Román-Portas y García, 2013: 134]), junto a las ruinosas inversiones de estas empresas al crear sus propios canales de televisión, produjeron graves pérdidas económicas y numerosos despidos, los cuales para estas empresas fueron culpa de la crisis financiera (Román-Portas y García, 2013: 126).

Con la aparición de las nuevas tecnologías de la comunicación a finales del anterior siglo, la competencia en el sector aumentó considerablemente, reduciéndose la relevancia de los periódicos impresos de forma paulatina. Si a esta revolución digital añadimos, como comenta Sotelo (2015: 848), los nuevos hábitos de los consumidores y los desastrosos efectos de la crisis económica en el sector de los medios de comunicación, el panorama actual para crear un periódico impreso en España no es nada halagüeño (Arranz, 2016b: 1).

La decadencia de la prensa impresa española es evidente, pues si se compara las actuales cifras de difusión y venta de ejemplares de los periódicos impresos españoles de tirada nacional más importantes (Tejón, 2016; Cano, 2017) con los datos que estos mismos medios de comunicación conseguían antes de la crisis económica iniciada en el 2008 (Redacción PRNoticias, 2015), se puede observar que el periódico en papel, con el paso del tiempo, se está convirtiendo en un producto que se dirige inexorablemente hacia la irrelevancia mediática y económica, pues el ciudadano español cada vez está menos interesado en adquirir este tipo de productos, al no encontrarse ninguna mejoría en las cifras de venta de los diarios impresos a pesar de la mejoría económica de los últimos años, es más, ni siquiera se vislumbra una cierta estabilidad en la demanda para este mercado (Tabla 1).

La grave caída en el número de ejemplares vendidos y en los ingresos monetarios recibidos por publicidad, son la causa principal por la terrible situación en la que se encuentra la prensa impresa generalista, deportiva y económica española, ingresando este tipo de prensa en el año 2016, 1.400 millones de euros menos que hace diez años por publicidad (Arranz, 2016b: 3), reduciendo costes el sector mediante aumentos progresivos del precio de los diarios, la disminución en el número de sus páginas y la reducción de su masa laboral. 
Tabla 1. Ventas de ejemplares de periódicos impresos de tirada nacional. Fuente: Redacción PRNoticias (2015) y Cano (2017)

\begin{tabular}{|l|l|l|l|l|}
\hline \multicolumn{6}{|l|}{ Ventas de ejemplares de periódicos (OJD) } \\
\hline Año & El País & ElMundo & Abc & La Raqón \\
\hline 2016 & 117.888 & 72.712 & 65.695 & 48.434 \\
\hline 2015 & 136.835 & 89.180 & 76.208 & 53.282 \\
\hline 2014 & 153.570 & 108.690 & 92.453 & 54.812 \\
\hline 2013 & 172.429 & 132.795 & 100.900 & 56.914 \\
\hline 2012 & 200.220 & 154.628 & 118.865 & 58.230 \\
\hline 2011 & 239.257 & 175.980 & 127.267 & 67.934 \\
\hline 2010 & 250.383 & 185.822 & 132.423 & 79.566 \\
\hline 2009 & 285.520 & 199.686 & 135.300 & 85.900 \\
\hline 2008 & 332.808 & 225.206 & 136.158 & 106.495 \\
\hline 2007 & 341.425 & 238.249 & 127.858 & 106.911 \\
\hline
\end{tabular}

A pesar de este sombrío panorama económico, en las última décadas en España, hubo varios intentos que apostaban en crear nuevos productos periodísticos de tirada nacional difundidos en papel que permitieran abarcar nuevos nichos de mercado, siendo los fiascos empresariales más relevantes la aparición en el año 1991 del primer tabloide sensacionalista español, el diario Claro (propiedad de Prensa Española [antiguo grupo editor del $A b c$ y el grupo alemán Alex Springer), el cual cerró a los cuatro meses de vida (González-Díez et al., 2015: 867); la aparición en el año 2007 del diario progresista Público (periódico vinculado al empresario Jaume Roures), medio que cerraría en el año 2012 (Fernández-Sande, 2013: 22) o la reconversión del periódico económico La Gaceta de los Negocios a diario generalista de ideología derechista en el año 2009 (Fernández-Sande, 2013: 26) propiciada por el Grupo Intereconomía, el cual también echaría el cierre en el 2013.

En el año 2015 aparecía el semanario Ahora, un medio dirigido por el periodista Miguel Ángel Aguilar (director de Diario 16 en el período 19771980), que pretendía diferenciarse de sus competidores digitales más recientes (Voz Pópuli [diario surgido en el 2011 de la mano de Jesús Cacho], El Español [fundado por Pedro José Ramírez en el año 2015], Ok Diario [creado por Eduardo Inda ese mismo año] o El Independiente [diario aparecido en el 2016 y dirigido por Casimiro García-Abadillo]) ofreciendo al interesado lector análisis más profundos y detallados que la que ofrecía la prensa digital española "sin dejar que la actualidad tergiverse la realidad subyacente" (Aguilar, 2015: 3), presentándose también el producto en papel. Su presidente editor justificaba así el medio impreso (Aguilar, 2015: 2): 
Por todas partes han cundido los pronósticos que aseguran la muerte del periodismo, de modo que en adelante su ejercicio sería asumido como una nueva dimensión por los ciudadanos. Pero la función de los periódicos impresos que presentan una versión organizada y ponderada del alud informe de la actualidad facilita una visión más comprensible que la aportada por el flujo incesante de la red. Así, la jerarquía de los medios de comunicación pareciera ser inversa a su multiplicación. Los lectores dictarán su fallo inapelable sobre si prevalecerá la cultura del todo gratis, que enriquece a los agregadores que canibalizan las aportaciones originales y pauperizan a los medios que se esfuerzan en conseguirlas mientras sitúan a los periodistas como especie a extinguir.

Esta defensa por el periódico impreso frente la inmediatez de la noticia digital, no sería la única razón por la que se creó este medio de comunicación, pues como comentaba Fernández-Sande (2013: 26) respecto la oportunidad de negocio que tenía el diario Público (y nosotros equiparamos también al semanario Ahora) al haber un único diario nacional de centro-izquierda en España, se interpretó erróneamente que había mercado para más periódicos de esta ideología al poderse ganar el favor de los lectores progresistas descontentos con la línea editorial de El País. No se debe olvidar que el director de Ahora, fue un trabajador histórico durante décadas del susodicho diario, mostrando públicamente sus quejas y diferencias con la dirección del grupo mediático y del periódico en sus últimos años como empleado, para finalmente prescindir El País de sus servicios el mismo año en el que Aguilar fundó su semanario (El Mundo, 2015: 1); si bien siguió colaborando en la Ser, cadena de radio propiedad del mismo grupo empresarial.

El cambio en la línea editorial de El País se hizo más evidente a partir de la crisis del 2008, dónde un debilitado económicamente Grupo Prisa tuvo que convivir con la primera legislatura (por mayoría absoluta) de Mariano Rajoy y el conservador Partido Popular (Alonso, 2015: 97); llegando a afirmar Antonio Caño (nombrado director de El País en 2014), que el periódico ya no era afín al Partido Socialista (Teruel, 2016: 215). Esta aproximación ideológica al centro-derecha del periódico se acentuaría más con la crisis interna del socialismo español, terminando El País por defender un gobierno en minoría del Partido Popular después de las elecciones del año 2016 (El País, 2016: 2), por lo que se pensaba que un periódico de izquierdas y totalmente ajeno a los intereses de los círculos mediáticos más próximos del ex-presidente Zapatero y del Grupo Prisa, como Ahora, podría tener cabida en el mercado español. 


\section{Metodología}

Para poder comprobar la relevancia que tuvo el semanario Ahora, mediante el análisis cualitativo de contenido se han examinado las noticias que aparecieron en los medios digitales de España acerca este producto periodístico, por medio de una revisión hemerográfica; evitándose usar la entrevista personal como técnica de recogida de información por considerarla poco fiable debido a la falta de autocrítica en declaraciones públicas de personas asociadas a este proyecto periodístico (Ahora Semanal, 2016c: 7). Para realizar tal labor, se usó el buscador Web de noticias de Google, siendo los descriptores utilizados 'Ahora' y 'Miguel Ángel Aguilar' y la acotación temporal elegida, el propio ciclo de vida del periódico. Esta sencilla búsqueda permitió recoger diferentes artículos periodísticos, los cuales, a modo de resumen, se han clasificado en función de su temática de la siguiente manera:

- Noticias relacionadas con el despido de Miguel Ángel Aguilar como columnista del diario El País y críticas de este periodista hacia la trayectoria del Grupo Prisa.

- Noticias asociadas con la aparición del semanario Ahora, su cierre y los motivos de su efímera existencia.

- Informaciones exclusivas de Ahora, recogidas en otros medios digitales.

De todos los enlaces encontrados, se han escogido únicamente noticias firmadas por periodistas de determinados diarios digitales españoles, en función de su similitud ideológica con el periódico analizado, la coincidencia temporal con otros proyectos empresariales parecidos y su prestigio o importancia en el sector, haciendo hincapié en las informaciones presentadas por su competidor El País, periódico en el que había trabajado el presidente editor de Ahora hasta la aparición del nuevo semanario.

También se han tenido en cuenta para la realización de este artículo, la propia presentación que hizo el semanario de sí mismo y del grupo de trabajadores que estaban detrás del proyecto empresarial en el primer número del periódico de la mano de su presidente editor, así como de las propias noticias del medio que informaban de la disolución de la empresa editora de Ahora y la despedida final que este periódico hizo de sus lectores. 


\section{Resultados}

Ahora se presentaba a sus lectores como una iniciativa ciudadana de variada procedencia (Ahora Semanal, 2015: 1) que acogía en sus páginas al pensamiento ilustrado, racional y progresista (Ahora Semanal, 2015: 3). Arropado el día de su presentación pública por una nutrida representación de cargos institucionales, políticos y personajes de la cultura (Intxausti, 2015: 3), la mayoría de medios de comunicación digitales dieron una aceptable acogida a este nuevo proyecto, reseñando a través de breves noticias de agencia, la aparición del semanario; mostrando la mayoría de periódicos digitales progresistas de España un mayor interés en el polémico despido de Aguilar de El País, pues el Grupo Prisa decidió que la actividad de Miguel Ángel Aguilar como su presidente editor y como columnista de opinión en El País eran situaciones incompatibles, habiendo decidido, después de unas declaraciones críticas del periodista sobre la situación del periódico de Prisa, no contar más con sus servicios (Galán, 2015: 6).

Esta situación fue aprovechada por el semanario para publicitarse y darse a conocer al público, pues su presidente editor (que también era tertuliano habitual de varios programas de televisión), pasó a ser noticia de los medios más progresistas, los cuales atacaban al Grupo Prisa por su supuesto viraje ideológico al conservadurismo; lo que permitió a Aguilar promocionar su semanario por los distintos medios de comunicación a los que asistía, promoción sencilla y económica que no triunfó, pues durante su corta vida, Ahora alcanzó una tirada de 15.000 ejemplares, de los que se vendían 5.000 (Arranz, 2016a: 6), cifras que hacían inviable la existencia de una publicación de tirada nacional en España.

Además de las noticias asociadas con la abrupta salida de Aguilar de El País (Soteras, 2015: 1) y el debate producido sobre la libertad existente en los medios de comunicación españoles por su corporativismo (Lardiés, 2015: 1), los medios digitales españoles solo hicieron referencia al semanario Ahora cuando informaron a sus lectores de las complicaciones sufridas inicialmente por Aguilar a la hora de crear su nuevo periódico (El Confidencial Digital, 2015: 1), cuando el productos se presentó en sociedad y a causa de su triste final, siendo muy puntuales los momentos en los que el periódico tuvo algo de notoriedad en los medios de comunicación, los cuales siempre estaban asociados con la actividad profesional de su hacedor, como por ejemplo, cuando su presidente editor protestó indignado por la impuntualidad del político Pablo Iglesias en una rueda de prensa para posteriormente abandonar 
la sala iracundo (Medialdea, 2016: 2) o cuando realizó furibundas críticas al antiguo diario en el que escribía (Cabanillas, 2016: 5).

El semanario ambicionaba convertirse en El País de finales de siglo XX (Piqué, 2016: 3), un periódico que se posicionara contra la política conservadora del Partido Popular gracias a no tener ataduras de ningún tipo, considerando al presidente Rajoy como el símbolo máximo de la permisividad con la corrupción política española. En palabras del último editorial del semanario (Ahora Semanal, 2016d: 4):

Pensar que seguirá sin pasar factura ni tener consecuencias que máximos dirigentes del PP inseparables de Rajoy estén hoy sentados en el banquillo por los casos de las tarjetas black y de la Gürtel es insostenible a medio plazo. De esa corrupción trae causa la repugnancia que todos muestran a facilitar con su abstención la investidura de Rajoy y la dificultad de que haya una decisión unánime del comité federal sobre el voto de los diputados del Grupo Parlamentario Socialista en el Congreso. Rajoy se piensa más blindado en La Moncloa, pero resistir ahí seguirá agravando el horizonte penal que tiene delante.

La animadversión total a la continuidad del gobierno de Rajoy en España no era la única seña de identidad del periódico, pues Ahora también tuvo afilados dardos contra el Grupo Prisa por sus ataques a Pedro Sánchez, el secretario general del Partido Socialista, el cual dimitió el 1 de octubre del año 2016 de su cargo en una situación de grave crisis interna del socialismo español. En palabras del propio periódico (Ahora Semanal, 2016a: 1):

El bombardeo implacable sobre el secretario general del PSOE que la aviación gubernamental y las escuadrillas mediáticas a su servicio llevan a cabo desde las elecciones del 20 de diciembre, redoblado a partir del 26 de junio y del 25 de septiembre, pretendía la abstención y consiguiente investidura de Mariano Rajoy como presidente del Gobierno.

A pesar de que durante todo el año 2016 hubo un continuo interés en la sociedad española por la evolución de la situación política y sus novedades, el periódico pasaba desapercibido en los quioscos españoles (a pesar de un favorable contexto histórico y el gran tamaño físico del mismo) y después de producirse en la empresa dos ampliaciones de capital consideradas insuficientes por el consejo editorial de Ahora (Ahora Semanal, 2016b: 6); ni las insuficientes medidas tomadas para reducir los costes económicos (el periódico se distribuía en 8.000 quioscos de ciudades españolas de más de 8.000 habitantes [Intxausti, 2015: 5] ni el incremento de ingresos alcanzado los 
últimos meses de vida del medio, pudieron salvar el periódico (Ahora Semanal, 2016b: 3). Sin un atisbo de autocrítica ante el evidente fracaso del proyecto, su editora Papel Futuro 2014 S.L., anunciaba a mediados de octubre del año 2016 su cierre, auto-elogiando la calidad de su semanario (Ahora Semanal, 2016c: 7): Ni nostálgicos del papel ni profetas de su desaparición, con el formato de la prensa internacional de calidad, intentando ofrecer una visión ponderada, evitando que la actualidad tergiverse la realidad, sin abdicar de la oferta digital, en defensa del periodismo de pago que solo se vende a sus lectores, favorables a la apertura de un espacio de reflexión pausada, en contraste con el vértigo de fragmentos noticiosos envenenados de sectarismo, convencidos de que sin articulación del debate público las libertades se degradan.

Este profundo orgullo por un periódico de tan corta vida y tan ruinoso económicamente, quedó bien descrito en palabras de Miguel Ángel Aguilar, dónde resaltó que Ahora será recordado en el futuro como un prestigioso periódico que no hizo concesiones ni al amarillismo ni al sectarismo (Ahora Semanal, 2016b: 8), si bien es interesante recordar que fue el propio Aguilar, a través de un medio digital competidor, el que señaló las fallas de su creación (Piqué, 2016: 5):

La vida y la muerte de Ahora dejan al menos tres lecciones, según Aguilar. Primera: hace falta dinero en serio y gastar fuerte en marketing. Además, la tendencia firme a los buenos resultados debe aparecer en un año o poco más. Segunda: es imposible rehuir "los asuntos calientes", como los llama, que conforman las tertulias de teles y radios y las conversaciones de la calle, por muy poco sustanciales que se los considere. Tercera: es preciso aglutinar esfuerzos periodísticos, ahora dispersos en una colección de cabeceras pequeñas que concentran talento, pero centrifugan recursos.

Las alabanzas por el difunto periódico no se verían reflejadas en el resto de medios, limitándose muchos diarios digitales a recoger (otra vez) breves notas de agencias informativas que anunciaban la desaparición del periódico, recalcándose también a algunas figuras que sustentaron el fallido proyecto (Forcada, 2016: 2); siendo llamativo que en esta ocasión, la edición digital de El País ni siquiera hubiera recogido la noticia del cese de actividad del negocio de su antiguo columnista; y si bien gran parte de los autores de los textos analizados por este estudio confirmaban que Ahora era una mercancía bastante digna para ponerse a la venta en el mercado, esta supuesta calidad extrema de la que presumía su fundador, tampoco destacaba sobre el resto de periódicos, o incluso (como comenta el mismo Aguilar) al semanario le faltaba "entrar en temas periodísticos más... calientes" (Piqué, 2016: 10); por lo que su precio de venta, muy superior a los diarios generalistas, y su tamaño en número de 
páginas mucho más reducido que la oferta de la competencia, no se justificaba en aras de esa autoproclamada brillantez periodística (Lamata, 2016: 8).

\section{Discusión}

Miguel Ángel Aguilar, histórico periodista progresista, poco conforme por el giro ideológico del periódico El País, fue el que decidió retomar el proyecto de desbancar del sector de la izquierda al antiguamente hegemónico diario de Prisa, diferenciándose del defenestrado diario Público en los siguientes aspectos:

- El nuevo periódico no sería de publicación diaria sino que estaría en los quioscos una vez por semana, lo que permitiría reducir costes, pues según Larrañaga (2009: 65), el gasto más importante de un periódico impreso es el uso de papel seguido de la partida y aprovisionamiento de otros consumos.

- Ahora se dirigía a un objetivo comercial distinto de los lectores de izquierda españoles (Público estaba orientado a un público mucho más joven y más progresista, mientras que Ahora se quería acercar al lector de mediana edad de centro-izquierda que aún compraba prensa en papel).

- Detrás de este nuevo proyecto no había ningún grupo empresarial, pues Ahora salió a la calle de la mano de centenares de inversionistas y todos tenían el compromiso de no superar el 10\% del capital de la empresa editora (Lamata, 2016: 3).

El proyecto de Ahora se defendió desde sus orígenes como un periodismo de reflexión y análisis frente a la inmediatez de las noticias de Internet y las redes sociales, por lo que es evidente que este producto no pretendía luchar en el competitivo mundo de los periódicos digitales, sino que su rival a batir más evidente era el diario El País, el cual estaba en un momento de debilidad económica y de prestigio muy grave, situación que conocía sobradamente Miguel Ángel Aguilar y el propio grupo Prisa. En palabras de Galán (2015: 6):

En las semanas previas a la aparición de ese periódico, se le comunicó a Aguilar que su participación en ese proyecto era incompatible con la continuación de sus colaboraciones en EL PAÍS, puesto que ambos medios eran competencia. Posteriormente, el propio Aguilar confirmó esa rivalidad en una comunicación interna a los socios de Ahora en la que les manifestaba que su periódico venía a llenar un hueco existente en España, donde, según sus 
palabras textuales, no había en este momento ningún periódico 'que se pudiera llevar con orgullo bajo el brazo'.

Es evidente que a pesar de que los creadores de Abora no se veían a sí mismos ni como "nostálgicos del papel ni profetas de su desaparición" (Ahora Semanal, 2016c: 7), nosotros consideramos que ésta es la descripción más clara y concisa que se puede hacer del difunto semanario, siendo el ejemplo más evidente de cómo un periódico en papel puede arruinar un periódico digital y los puestos de trabajo asociados a esta labor, el caso del diario regional Xornal de Galicia (cuyo propietario era el grupo constructor San José), el cual fue editado a finales del año 2008 y desapareció tres años más tarde; lo que provocó que su hermano digital Xornal.com, de mayor antigüedad, también tuviera que cerrar un mes después del diario impreso (Europa Press, 2011: 8).

Es evidente por lo tanto, que las mayores debilidades del proyecto empresarial del semanario Ahora (entre otros factores) fueron las actividades asociadas con la prensa impresa, considerando las trabas más importantes para el éxito del periódico las siguientes:

- Excesivos costes en difundir un producto periodístico físico a nivel nacional con tan limitada tirada, siendo excesivamente más caro la presencia del medio en las regiones insulares del país (Arranz, 2014: 1).

- Precio poco competitivo ( $3 €)$ para un producto que se da a conocer al mercado [compárese el precio que tenía Abora con los precios iniciales de los diarios en papel La Razón (El País, 1998: 6) y Público (Casanueva, 2007: 6)].

- Híper-liderazgo de su presidente editor frente el rol del director del medio, identificando la sociedad al periodista Miguel Ángel Aguilar pero no al semanario.

- Inexistencia de un grupo empresarial que sustentase económicamente al periódico (Lamata, 2016: 3).

- Caída de ingresos generalizada por publicidad en el sector de la prensa impresa (Arranz, 2016b: 3).

A manera de hipótesis que ha surgido del presente estudio y que se pretende comprobar en estudios subsecuentes, si el semanario se ofreciera como periódico gratuito, tampoco creemos que la situación mejorara lo suficiente para garantizar su viabilidad empresarial, pues este tipo de medios también están en completa decadencia, ya que "el soporte de prensa gratuita resultó uno de los más afectados por la crisis económica" (Román-Portas y García, 
2013: 134). Además la empresa editora de Abora siempre se consideró contraria a la prensa gratuita ya fuera en papel o digital, pues ellos mismos se presentaban como los adalides de la "defensa del periodismo de pago que solo se vende a sus lectores" (Ahora Semanal, 2016c: 7).

Aguilar, como periodista de la vieja guardia, es muy crítico con el periodismo actual, donde la inmediatez de la noticia y su impacto es más importante que la investigación exhaustiva o la redacción y presentación de la misma. Este nuevo estilo, en el que las redes sociales tienen una importancia vital, está profundamente enraizado con el denominado periodismo ciudadano, una nueva forma de comunicación, en la que cualquier individuo, con el uso de las nuevas tecnologías puede ser considerado como un comunicador social (Martínez-Arias, 2015: 111). Esta nueva forma de entender el periodismo ha permitido conectar a los más jóvenes con los hábitos de consumo de información; pues en los últimos años; el demandante de información es una persona joven, inquieta y culta (Meso, 2005 citado en Martínez-Arias, 2015: 110), algo de lo que el semanario en papel Ahora estaría muy alejado (Piqué, 2016: 3).

Estos males del periodismo que denunciaba Aguilar, el cual consideraba una disminución en la calidad de la información, además de ser una amenaza a la profesión periodística (Aguilar, 2015: 2), se podrían resumir perfectamente en las siguientes palabras de Carrasco (2010, citado en Martínez-Arias, 2015: 110):

Existen millones de páginas informativas, que no periodísticas, y los periodistas profesionales sólo hacen una pequeña parte de esos contenidos. ¿Quién los escribe? La sociedad de la información está en otras manos, ya que no es dominio exclusivo de las empresas. Las empresas informativas están en crisis y buscan fórmulas para salir de ella y una de sus estrategias implica el involucrar a los lectores como parte del proceso informativo.

En definitiva, un mercado como el de la prensa impresa, con unos elevados costes fijos que la prensa digital no tiene, como por ejemplo "los costes de impresión, distribución, contra-distribución (debidos a la devolución de los diarios no vendidos) y almacenamiento de ejemplares" (Albornoz, 2006 citado en Larrañaga, 2009: 70), donde los ingresos publicitarios se desploman anualmente y éstos se dirigen hacia Internet (Arranz, 2016b: 4), junto a una atroz competencia entre los periódicos de papel que todavía existen, no invitan al optimismo; teniendo que asumir los inversores de Abora cuantiosas pérdidas económicas por un anticuado producto periodístico de lujo. 


\section{Conclusiones}

Se podría comparar al periódico impreso Ahora con un telegrama, ya que éste era un producto anacrónico y desfasado antes de que saliera su primer número al mercado, por lo que no tenía ningún futuro a nivel económico. Es evidente, pues la realidad habla por sí misma, que el objetivo de crear un nuevo referente mediático en la izquierda española, que fuera más combativo que el grupo Prisa contra la ideología conservadora y liberal, pero sin caer en las redes mediáticas de otras formaciones políticas de izquierda (como el partido político Podemos y sus confluencias) no tuvo éxito.

El descalabro del proyecto encabezado por Miguel Ángel Aguilar es más doloroso si se compara con los proyectos empresariales que dirigen compañeros periodistas de su generación como Pedro José Ramírez o Casimiro García-Abadillo u otros más jóvenes como Ignacio Escolar, diarios que siguen en activo a inicios del año 2017, teniendo únicamente en común que todos ellos han apostado exclusivamente por la prensa digital y rechazado la prensa impresa en papel.

Con el cierre de Ahora, el fallo de los lectores se hizo público, y parafraseando a su presidente editor, parece ser que al final ha prevalecido la cultura caníbal del todo gratis, habiéndose hundido estrepitosamente las aportaciones originales (Aguilar, 2015: 2). Aunque Aguilar advertía tras el final de Ahora que no se recibían condolencias (Ahora Semanal, 2016b: 8), sinceramente creemos que el epitafio para esta publicación bien puede ser el certero resumen realizado por Piqué (2016: 4):

El semanario ha sido la carga de la Brigada de caballería de Pomerania contra el batallón de la $20^{\circ}$ de Infantería Motorizada de la Wehrmacht sorprendido en el río Brda. Es muy posible que sea la última iniciativa impresa de España en su género. Una redacción de una veintena de personas, entre ellas una docena de periodistas, editaba una versión digital y otra impresa, que vendía en quiosco unos 5.000 periódicos y sumaba 1.200 suscriptores. Entre una y otra, Ahora ha facturado este año cerca de 180.000 euros en publicidad. No son cifras malas. Son peores.

\section{Bibliografía}

Aguilar, M. A. (2015). AHORA se presenta y dice. Ahora, 1, Madrid, septiembre. Recuperado de https://www.ahorasemanal.es/ahora-se-presentay-dice: 
Ahora Semanal (2015). Quiénes somos. Ahorasemanal.es. Recuperado de https://www.ahorasemanal.es/quienes-somos Fecha de consulta: 02/01/2017

_ (2016a). Guerra civil en el PSOE. Ahora, 53, Madrid, septiembre.

Recuperado de https://www.ahorasemanal.es/guerra-civil-en-el-psoe Fecha de consulta: 10/01/2017

_ (2016b). Disolución de la sociedad y cierre del periódico AHORA. Ahora, 54, Madrid, octubre. Recuperado de https://www.ahorasemanal.es/disolucion-de-la-sociedad-y-cierre-delperiodico-ahora Fecha de consulta: 17/01/2017

_ (2016c). AHORA se despide de ustedes. Ahora, 55, Madrid, octubre.

Recuperado de https://www.ahorasemanal.es/ahora-se-despide-de-ustedes-

_ (2016d). Sin condiciones, ¿ $\sin$ consecuencias? Ahora, 55, Madrid, octubre. Recuperado de

https://www.ahorasemanal.es/sin-condiciones, - sin-consecuencias

Alía, F. (2013). La prensa histórica española en Internet. Historiografías, 6, juliodiciembre, pp.104-123.

Almirón, N. (2007). La deuda de Sogecable y Prisa: análisis y génesis de una estrategia empresarial global de alto riesgo. Quaderns del CAC, 29, pp. 105-116.

Alonso, M. (2015). Influencias políticas y empresariales en la prensa española. El cambio de dirección en El País. Revista de Comunicación de la SEECI, 19(38), pp. 92-113.

Arranz, R. (2014). ABC dejará de publicarse en papel en Baleares y Canarias en 2015, y sólo podrá comprarse en Kiosko y Más. Vozpopuli.com. Recuperado de http://www.vozpopuli.com/medios/ABC-Prensa-Vocento-periodicoCanarias-Baleares-Islas-distribucion-impresion-gastos-recortes-ajustesprensa_0_758324204.html Fecha de consulta 28/02/2017

_ (2016a). Miguel Ángel Aguilar cierra su semanario 'Ahora': "Hemos consumido todos los recursos". Vozpopuli.com. Recuperado de http:/ /www.vozpopuli.com/medios/Miguel-Angel-Aguilarsemanario_0_960204525.html Fecha de consulta: 29/12/2016 
_ (2016b). Cinco años de pesadilla para el papel: los grandes periódicos pierden 500.000 ejemplares. Vozpopuli.com. Recuperado de http://www.vozpopuli.com/medios/pesadilla-principales-periodicos-pierdenejemplares 0 984802368.html Fecha de consulta: 29/12/2016

Cabanillas, A. (2016). Miguel Ángel Aguilar carga contra 'El País' y Vargas Llosa: "Es una situación penosa". Elmundo.es. Recuperado de http://www.elmundo.es/television/2016/07/04/577ab031e5fdeaa5198b4659 .html Fecha de consulta: 17/01/2016

Cano, F. (2017). OJD 2016: 'El País', 'El Mundo' y 'ABC' pierden otros 61.000 ejemplares de difusión. PRNoticias.com. Recuperado de http://prnoticias.com/prensa/difusion-ojd/20159826-ojd-el-pais-el-mundo-yabc-pierden-otros-61-000-ejemplares Fecha de consulta 28/02/2017

Casanueva, M. (2007). El diario 'Público' nace sin editoriales. Elmundo.es. Recuperado de http://www.elmundo.es/elmundo/2007/09/20/comunicacion/1190308120. $\underline{\mathrm{html}}$

De Aguinaga, E. (2002). El periodista en el umbral del siglo XXI. Estudios sobre el Mensaje Periodístico, 8, pp. 157-170.

El Confidencial Digital (2015). Miguel Ángel Aguilar retrasa el lanzamiento del semanario 'Ahora' a otoño. Elconfidencialdigital.com. Recuperado de http://www.elconfidencialdigital.com/medios/Miguel-Angel-Aguilarlanzamiento-Ahora 0 2505349461.html Fecha de consulta: 14/01/2016

El Mundo (2015). 'El País' despide a Miguel Ángel Aguilar por criticarlo en 'The New York Times'. Elmundo.es. Recuperado de http://www.elmundo.es/television/2015/11/11/56436cdd46163552598b45a 7.html Fecha de consulta: 17/01/2016

El País (1998). "La Razón" se proclama apartidista y defensor de la unidad de España. El País, Madrid, 7.845, noviembre. Recuperado de http://elpais.com/diario/1998/11/06/sociedad/910306815_850215.html Fecha de consulta: 28/02/2017

_ (2016). La deriva de Sánchez. El País, 14.325, Madrid, septiembre.

Recuperado de http://elpais.com/elpais/2016/09/22/opinion/1474568984 419976.html Fecha de consulta: 10/01/2016 
El Nacional (2016). Aguilar asegura que Interior pagó 1,5 MEUR a un confidente por información sobre Trias. Elnacional.cat. Recuperado de http://www.elnacional.cat/es/politica/aguilar-interior-pago-confidentecuentas-trias 110713 102.html Fecha de consulta: 28/02/2017 Europa Press (2011). Xornal.com decide cerrar un mes después de desaparecer la cabecera impresa y 40 profesionales perderán su trabajo. Elpaís.com. Recuperado de http://elpais.com/elpais/2011/09/19/actualidad/1316420233 850215.html Fecha de consulta: 05/01/2016

Fernández-Sande, M. (2013). La crisis de la prensa en España: análisis del caso Público, un diario nacido a contracorriente. Revista de Comunicación Vivat Academia, 15(122), pp. 22-39.

Forcada, D. (2016). Miguel Ángel Aguilar cierra 'Ahora', el último intento de sacar otro periódico de papel. Elconfidencial.com. Recuperado de http://www.elconfidencial.com/comunicacion/2016-10-07/miguel-angelaguilar-cierra-ahora-el-ultimo-intento-de-sacar-otro-periodico-depapel 1271880/Fecha de consulta 07/03/2017

Galán, L. (2015). El final de una colaboración. Elpaís.com. Recuperado de http://elpais.com/elpais/2015/11/14/opinion/1447506543 884985.html Fecha de consulta: 17/01/2016

García-Galindo, J. (2005). Estudios de periodismo. Los primeros tratadistas españoles. En J. M. Desvois (coord.) Prensa, impresos, lectura en el mundo hispánico contemporáneo: homenaje a Jean-François Botrel (pp. 179-192). París: Presse, Imprimés, Lecture dans l'Aire Romane.

González-Díez, L., Puebla-Martínez, B., Birkner, T. y Pérez-Cuadrado, P. (2015). El diseño periodístico como actor fundamental en los modelos de prensa popular. Una aproximación a las causas del cierre del diario Claro. Revista Latina de Comunicación Social, 70, pp. 859-877.

Intxauti, A. (2015). AHORA, un periódico con edición semanal en papel. Elpais.com. Recuperado de http://cultura.elpais.com/cultura/2015/09/18/actualidad/14425809392543 07.html Fecha de consulta: 17/01/2016

Lamata, J. F. (2016). Miguel Ángel Aguilar cierra "Ahora” y demuestra que no es fácil ser un Polanco. Esdiario.com. Recuperado de 
http://www.esdiario.com/203660745/Cierre-de-Ahora-La-utopia-de-unperiodista-luchando-contra-los-numeros.html Fecha de consulta: 13/01/2016

Lardiés, A. (2015). Prisa y el resto de editores atacan a 'The New York Times'. Elespañol.com. Recuperado de http://www.elespanol.com/espana/20151112/78742163 0.html Fecha de consulta: 28/02/2017

Larrañaga, J. (2009). La crisis del modelo económico de la industria de los periódicos. Estudios Sobre el Mensaje Periodístico, 15, pp. 61-81.

Martínez-Arias, S. (2015). Periodismo ciudadano, en los límites de la profesión periodística. Estudios sobre el Mensaje Periodístico, 21, pp. 109-118.

Medialdea, S. (2016). El show de Pablo Iglesias con la prensa. Abc.es. Recuperado de http://www.abc.es/espana/abci-show-pablo-iglesias-prensa201604081508_noticia.html Fecha de consulta: 03/01/2016

Nogales, A. y Mancinas-Chávez, R. (2014). Los diarios de referencia en el mercado de la prensa española. Propiedad, grupos mediáticos y conexiones estructurales. Correspondencias y Análisis, 4, pp. 307-333.

Piqué, A. M. (2016). Aguilar: "En la política y los medios vuelven a repartirse las cartas". Elnacional.cat. Recuperado de http://www.elnacional.cat/es/cultura-ideas-artes/ahora-semanal-cierramiguel-angel-aguilar 114318 102.html Fecha de consulta: 28/02/2017 Redacción PRNoticias (2015). 'El País', 'El Mundo' y 'ABC' pierden la mitad de su difusión (461.000 ejemplares) durante la crisis. PRNoticias.com. Recuperado de http://prnoticias.com/prensa/difusion-ojd/20138014-el-paisel-mundo-y-abc-pierden-la-mitad-de-su-difusion-461-000-ejemplares-durantela-crisis Fecha de consulta: 17/01/2016

Sotelo, J. (2015). Dos grupos de comunicación en crisis y una fusión en ciernes: el caso de Unidad Editorial y Vocento. Opción, 31(6), pp. 835-851.

Soteras, J. (2015). 'El País' despide a Miguel Ángel Aguilar. Infolibre.es. Recuperado de http://www.infolibre.es/noticias/medios/2015/11/10/pais despide miguel angel_aguilar_40643_1027.html Fecha de consulta: 28/02/2017 
Román-Portas, M. y García, A. (2013). Crisis económica y grupos de comunicación en España (2008-2011). International Journal of Marketing, Communication and New Media, 1(1), julio-diciembre, pp. 124-147.

Tejón, C. (2016). OJD: Las ventas de 'El País' (-20\%) y 'El Mundo' (-19,2\%) se desploman en noviembre. PRNoticias.com. Recuperado de http://prnoticias.com/prensa/difusion-ojd/20159173-ojd-noviembre-ventasel-pais-el-mundo-desplome Fecha de consulta: 16/01/2016

Teruel, L. (2016). El impacto de la crisis política y económica sobre la polarización de los medios españoles. Historia y Comunicación Social, 21(1), pp. 203-220. 


\section{(c) (i) (2) \\ BY SA}

Licencia Creative Commons

Miguel Hernández Communication Journal

mhjournal.org

\section{Cómo citar este texto:}

Pedro Vázquez-Miraz (2017): "El fracaso del periódico Ahora, el ocaso de la prensa impresa española", en Miguel Hernández Communication Journal, nº, pp. 267 a 286. Universidad Miguel Hernández, UMH (Elche-Alicante). Recuperado el — de de 20_ de: [link del artículo en mhjournal.org] 